\title{
Preheating with extra dimensions
}

\author{
Shinji Tsujikawa * \\ Department of Physics, Waseda University, 3-4-1 Ohkubo, Shinjuku-ku, Tokyo 169-8555, Japan
}

(October 25, 2018)

\begin{abstract}
We investigate preheating in a higher-dimensional generalized Kaluza-Klein theory with a quadratic inflaton potential $V(\phi)=\frac{1}{2} m^{2} \phi^{2}$ including metric perturbations explicitly. The system we consider is the multi-field model where there exists a dilaton field $\sigma$ which corresponds to the scale of compactifications and another scalar field $\chi$ coupled to inflaton with the interaction $\frac{1}{2} g^{2} \phi^{2} \chi^{2}+\tilde{g}^{2} \phi^{3} \chi$. In the case of $\tilde{g}=0$, we find that the perturbation of dilaton does not undergo parametric amplification while the $\chi$ field fluctuation can be enhanced in the usual manner by parametric resonance. In the presence of the $\tilde{g}^{2} \phi^{3} \chi$ coupling, the dilaton fluctuation in sub-Hubble scales is modestly amplified by the growth of metric perturbations for the large coupling $\tilde{g}$. In super-Hubble scales, the enhancement of the dilaton fluctuation as well as metric perturbations is weak, taking into account the backreaction effect of created $\chi$ particles. We argue that not only is it possible to predict the ordinary inflationary spectrum in large scales but extra dimensions can be held static during preheating in our scenario.
\end{abstract}

\section{INTRODUCTION}

Physics in higher-dimensions has received much attention in an attempt to unify the interactions in Nature originating from Kaluza and Klein. For example, the ten-dimensional $E_{8} \times E_{8}$ heterotic superstring theory would be a strong candidate to describe the real world [1]. The strong coupling limit of this theory was found to be the eleven-dimensional supergravity [2], which is equivalent to the low-energy effective M-theory. Since the spacetime we recognize is four-dimensional, we conventionally utilize some mechanisms of dimensional reduction assuming that extra dimensions are compactified on some manifolds (Kaluza-Klein reductions). Recently, Randall and Sundrum [3] proposed an alternative way of compactifications based on brane models, which was originally introduced as a solution to the hierarchy problem between the weak and Planck scale [4,5]. In the brane scenario, gravity works in the five-dimensional bulk of spacetime while matter fields are confined in four-dimensions. In this paper, however, we adopt conventional Kaluza-Klein reduction from a higher-dimensional action whose dimension is larger than five.

One of the most important topics which plagues such higher-dimensional theories is the stability of extra dimensions. The internal dimensions need to be held static against small fluctuations in order to settle in the present universe which would be a direct product of the four-dimensional Minkowski spacetime $M^{4}$ and a small internal space $K^{d}$. In this respect, the basic argument is to introduce a cosmological constant in the higher-dimensional action and keep extra dimensions static with the existence of some fields. In such models including the Candelas-Weinberg (CW) model [6] (sphere compactification with a cosmological constant and the one-loop quantum correction), it has been recognized that the present vacuum is static against linear perturbations [7] and even non-linear large perturbations [8]. It was also found that stability of the internal space is preserved against a quantum tunneling without the topology change [9].

From a cosmological point of view, it is natural to ask whether the internal space is held static during an inflationary

\footnotetext{
*electronic address:shinji@gravity.phys.waseda.ac.jp
} 
epoch [10]. Amendola et al. [11] considered stability of compactification in the CW model with the existence of an inflaton field $\phi$. In old, new, and extended inflation models, the system exhibits semiclassical properties in which the stability is preserved as long as the transition probability for the scale of the internal space to tunnel through its potential barrier is smaller than that of inflaton. In the chaotic inflation model, the expansion of the internal space can be classically avoided if we choose the initial value of inflaton and parameters of the model appropriately. Amendola et al. also obtained the upper bound for the present scale of the internal space as $b_{*} \lesssim \sqrt{d} \times 10^{5} l_{\mathrm{pl}}$, where $l_{\mathrm{pl}}$ is the Planck length, by the requirement of successful chaotic inflation and stability of compactifications. As for the inflationary scenario in generalized Einstein theories including Brans-Dicke and induced gravity theories, Berkin and Maeda [12] analyzed the dynamics of inflation in new and chaotic inflationary models in the presence of a dilaton field $\sigma$ with a potential $U(\sigma)=0$. In the context of large internal dimensions, several authors [13] recently investigated inflation with the higher-dimensional fundamental Planck scale in the $\mathrm{TeV}$ region.

After the inflationary period ends, the system enters a reheating stage. It has been recognized that reheating will turn on by an explosive particle production which is called preheating 14 16. As compared with other inflationary models, the chaotic inflationary scenario typically leads to the strong amplification of a scalar field $\chi$ coupled to inflaton with the interaction $\frac{1}{2} g^{2} \phi^{2} \chi^{2}$ due to parametric resonance during the oscillating stage of inflaton. In this scenario, a lot of works have already been done using analytic approaches based on the Mathieu [15, 16] or Lamé equations [17] and numerical computations by mean field approximations [18 20] or full lattice simulations [21 23]. The existence of the preheating stage is important in the sense that it would affect the baryogenesis in grand unified scale [24], topological defect formation [25], nonthermal phase transition 26], and gravitational waves [27].

Recently, Mazumdar and Mendes [28] considered preheating in generalized Einstein theories including higherdimensional theories in the massive chaotic inflationary model $V(\phi)=\frac{1}{2} m^{2} \phi^{2}$ with a scalar field $\chi$ coupled to inflaton. After dimensional reductions, we have a dilaton field $\sigma$ which corresponds to the radius of extra dimensions. They investigated the multi-field system of scalar fields $\phi, \chi, \sigma$ in the case where dilaton does not have its own potential. It was pointed out that long-wave modes $(k \rightarrow 0)$ of the fluctuation of dilaton can be enhanced due to the growth of metric perturbations. Although their scheme is based on the torus compactifications which have zero curvature, compactifications on the sphere give rise to a potential term due to the curvature of the internal space. It is worth investigating whether the stability of compactification is preserved or not during preheating, when dilaton has its own potential. In this paper, we adopt the dilaton potential based on the CW model in the presence of a massive inflaton field, and analyze the evolution of scalar field fluctuations including the backreaction effect of created particles. We include metric perturbations explicitly for the evolution equations, and also investigate whether this model predicts the density perturbation observed by the Cosmic Background Explorer (COBE) satellite.

This paper is organized as follows. In the next section, we describe our model and consider the dynamics of inflation in the presence of the dilaton field. In Sec. III, we investigate the parametric amplification of field fluctuations during preheating including metric perturbations. It is particular of interest to study the evolutions of super-Hubble dilaton and metric perturbations. We present our conclusion and discussion in the final section. 


\section{INFLATION WITH EXTRA DIMENSIONS}

We investigate a model in $D=d+4$ dimensions with a cosmological constant $\bar{\Lambda}$ and a single scalar field $\bar{\phi}$,

$$
S=\int d^{D} x \sqrt{-\bar{g}}\left[\frac{1}{2 \bar{\kappa}^{2}} \bar{R}-2 \bar{\Lambda}+\overline{\mathcal{L}}(\bar{\phi})\right]
$$

where $\bar{\kappa}^{2} / 8 \pi \equiv \bar{G}$ and $\bar{R}$ are the $D$-dimensional gravitational constant and a scalar curvature with respect to the $D$-dimensional metric $\bar{g}_{M N}$, respectively. The Lagrangian density for the minimally coupled $\bar{\phi}$ field is written as

$$
\overline{\mathcal{L}}(\bar{\phi})=-\frac{1}{2} \bar{g}^{M N} \partial_{M} \bar{\phi} \partial_{N} \bar{\phi}-\bar{V}(\bar{\phi}),
$$

where $\bar{V}(\bar{\phi})$ is a potential of the $\bar{\phi}$ field.

We compactify extra dimensions to a $d$-dimensional sphere $S^{d}$. Then the metric $\bar{g}_{M N}$ is expressed as

$$
d s_{D}^{2}=\bar{g}_{M N} d x^{M} d x^{N}=\hat{g}_{\mu \nu} d x^{\mu} d x^{\nu}+b^{2} d s_{d}^{2}
$$

where $\hat{g}_{\mu \nu}$ is a four-dimensional metric, $b$ is a scale of the $d$-dimensional sphere whose present value is $b_{*}$, and $d s_{d}^{2}$ is a line element of the $d$-unit sphere. After dimensional reduction, the action (2.1) yields

$$
S=\int d^{4} x \sqrt{-\hat{g}}\left(\frac{b}{b_{*}}\right)^{d}\left[\frac{1}{2 \kappa^{2}}\left\{\hat{R}+d(d-1) \frac{\partial_{\mu} b \partial_{\nu} b}{b^{2}} \hat{g}^{\mu \nu}+\frac{d(d-1)}{b^{2}}\right\}+V_{d}^{0}\{\hat{\mathcal{L}}(\hat{\phi})-2 \bar{\Lambda}\}\right],
$$

where $\kappa^{2} / 8 \pi$ is Newton's gravitational constant which is expressed as $\kappa^{2} / 8 \pi=\bar{\kappa}^{2} /\left(8 \pi V_{d}^{0}\right)$ with the present volume of the internal space $V_{d}^{0}$, and $\hat{R}$ is a scalar curvature with respect to $\hat{g}_{\mu \nu}$. The action (2.4) is different from the form of the Einstein-Hilbert action due to the time-dependent term $\left(b / b_{*}\right)^{d}$. In order to obtain the usual form, we perform the following conformal transformation,

$$
\hat{g}_{\mu \nu}=\exp \left(-d \frac{\sigma}{\sigma_{*}}\right) g_{\mu \nu}
$$

where $\sigma$ is the so-called dilaton field which is defined by

$$
\begin{aligned}
\sigma & =\sigma_{*} \ln \left(\frac{b}{b_{*}}\right), \\
\sigma_{*} & =\left[\frac{d(d+2)}{2 \kappa^{2}}\right]^{1 / 2} .
\end{aligned}
$$

Then the four-dimensional action in the Einstein frame can be described as

$$
S=\int d^{4} x \sqrt{-g}\left[\frac{1}{2 \kappa^{2}} R-\frac{1}{2} g^{\mu \nu} \partial_{\mu} \sigma \partial_{\nu} \sigma-U(\sigma)-\frac{1}{2} g^{\mu \nu} \partial_{\mu} \phi \partial_{\nu} \phi-\exp \left(-d \frac{\sigma}{\sigma_{*}}\right) V(\phi)\right],
$$

where $R$ is a scalar curvature related with $g_{\mu \nu}$, and a scalar field $\phi$ is defined by $\phi=\sqrt{V_{d}^{0}} \hat{\phi}$. The potential $U(\sigma)$ for the $\sigma$ field is expressed as

$$
U(\sigma)=\frac{\bar{\Lambda}}{\kappa^{2}} e^{-d \sigma / \sigma_{*}}-\frac{d(d-1)}{2 \kappa^{2} b_{*}^{2}} e^{-(d+2) \sigma / \sigma_{*}} .
$$

The second term in Eq. 2.9) appears due to the curvature of the internal space by compactifications on the sphere $S^{d}$. However, since the potential (2.9) lacks a local minimum to stabilize the dilaton field, one needs to introduce quantum correction effects which are so-called Casimir effects. 
Adding a one-loop effective action which is proportional to $e^{-2(d+2) \sigma / \sigma_{*}}$ to the potential $U(\sigma)$ and imposing the conditions that the $\sigma$ field has a local minimum at $\sigma=0$ and its extremum is zero, $U(\sigma)$ can be written in the following form [29]:

$$
U(\sigma)=\alpha\left[\frac{2}{d+2} e^{-2(d+2) \sigma / \sigma_{*}}+e^{-d \sigma / \sigma_{*}}-\frac{d+4}{d+2} e^{-(d+2) \sigma / \sigma_{*}}\right]
$$

with

$$
\alpha=\frac{d(d-1)(d+2)}{2 \kappa^{2}(d+4) b_{*}^{2}} .
$$

The first, second, and third terms in (2.10) are due to the Casimir energy, the cosmological constant, and the curvature of the internal space, respectively. The potential $U(\sigma)$ in the action (2.8) has a local minimum at $\sigma=0$ and a local maximum at $\sigma_{c}(>0)$ which depends on the extra dimension $d$. In order to reach the final state $\sigma=0$ which corresponds to the present scale of the internal space $b=b_{*}$, the initial value of $\sigma$ is required to be $0<\sigma_{I}<\sigma_{c}$ (we assume $\sigma_{I}>0$ ), where the subscript $I$ denotes the initial value. Then $\sigma$ evolves toward the minimum of its potential, and begins to oscillate around $\sigma=0$. Since extra dimensions are compactified on the sphere, this gives rise to the four-dimensional Kaluza-Klein field $\psi_{l m}$ whose mass is given by $M_{l}^{2}=l(l+d-1) e^{-(d+2) \sigma / \sigma_{*}} / b_{*}^{2}$. It was suggested in Ref. [29] that Kaluza-Klein modes can be excited by the oscillation of the $\sigma$ field in the flat Friedmann-RobertsonWalker (FRW) background. Later, we found that catastrophic enhancement of Kaluza-Klein modes does not occur relevantly for any values of $\sigma_{I}$ and the quantum number $l \geq 1$ [30]. Hence we only consider the case of $l=0$ in this paper. For a complete study, however, we have to take into account the existence of Kaluza-Klein modes with $l \geq 1$.

In the presence of the inflaton field $\phi$, the effective potential for the dilaton field is described by the action (2.8) as follows

$$
U_{1}(\sigma, \phi)=\alpha\left[\frac{2}{d+2} e^{-2(d+2) \sigma / \sigma_{*}}+e^{-d \sigma / \sigma_{*}}\left\{1+\frac{V(\phi)}{\alpha}\right\}-\frac{d+4}{d+2} e^{-(d+2) \sigma / \sigma_{*}}\right] .
$$

The stability of compactification during inflation with a potential (2.12) in several models of inflation was analyzed in Ref. [11]. Since we are interested in the model where strong parametric amplification of scalar fields can be expected during preheating, we adopt the quadratic potential of chaotic inflation,]

$$
V(\phi)=\frac{1}{2} m^{2} \phi^{2} .
$$

In Fig. 1, we depict the effective potential (2.12) with (2.13). As was pointed out in Ref. [11], $U_{1}(\sigma, \phi)$ has either two local extrema or no local extrema for a fixed value of $\phi$. When $\phi$ is smaller than some critical value $\phi_{c}$, the potential barrier which prevents the $\sigma$ field from going toward infinity exists, and the scalar field evolves toward the potential minimum at $\phi=\sigma=0$. However, when $\phi>\phi_{c}$, this barrier disappears and the internal space grows without limit. The critical value $\phi_{c}$ can be obtained by solving the equation $\partial U_{1} / \partial \sigma=\partial^{2} U_{1} / \partial \sigma^{2}=0$ as

$$
\phi_{c}^{2}=\frac{2 \alpha}{m^{2}}\left[\left(1+\frac{2}{d}\right)\left(\frac{1}{2}\right)^{2 /(d+2)}-1\right] .
$$

\footnotetext{
${ }^{1}$ In the model of the self-coupling potential $V(\phi)=\frac{1}{4} \lambda \phi^{4}$, we will give some discussions in the final section.
} 
In order to result in the present vacuum $\sigma=0$, the inflaton $\phi$ is constrained as

$$
\phi^{2}<\phi_{c}^{2}=\frac{d(d-1)(d+2)}{8 \pi(d+4)}\left[\left(1+\frac{2}{d}\right)\left(\frac{1}{2}\right)^{2 /(d+2)}-1\right]\left(\frac{m_{\mathrm{pl}}}{m}\right)^{2} \frac{1}{b_{*}^{2}},
$$

where we have used Eq. (2.11). In the chaotic inflationary scenario, the initial value of inflaton is required to be $\phi_{I} \gtrsim 3 m_{\mathrm{pl}}$ in order to obtain the number of e-foldings greater than 60 . Further, the density perturbation observed by the COBE satellite constrains the coupling of inflaton as $m \sim 10^{-6} m_{\mathrm{pl}}$. Then the condition (2.15) leads to the following bound for the present value of the internal space:

$$
b_{*}^{2} \lesssim \frac{d(d-1)(d+2)}{72 \pi(d+4)}\left[\left(1+\frac{2}{d}\right)\left(\frac{1}{2}\right)^{2 /(d+2)}-1\right] \frac{10^{12}}{m_{\mathrm{pl}}^{2}} .
$$

For example, when $d=2$ and $d=6, b_{*} \lesssim 5 \times 10^{4} / m_{\mathrm{pl}}$ and $b_{*} \lesssim 1.1 \times 10^{5} / m_{\mathrm{pl}}$, respectively. For large values of $d$, Eq. (2.16) reads

$$
b_{*} \lesssim \sqrt{d} \times 10^{5} / m_{\mathrm{pl}}
$$

As was suggested in Ref. [11], this value is by about ten orders of magnitude smaller than the experimental bound $b_{*} \lesssim 10^{17} / m_{\mathrm{pl}}$. It is worth mentioning that such theoretical bound is derived by the analysis of stability of compactification during inflation. In what follows, we use the values of $b_{*}$ which satisfy the relation (2.16).

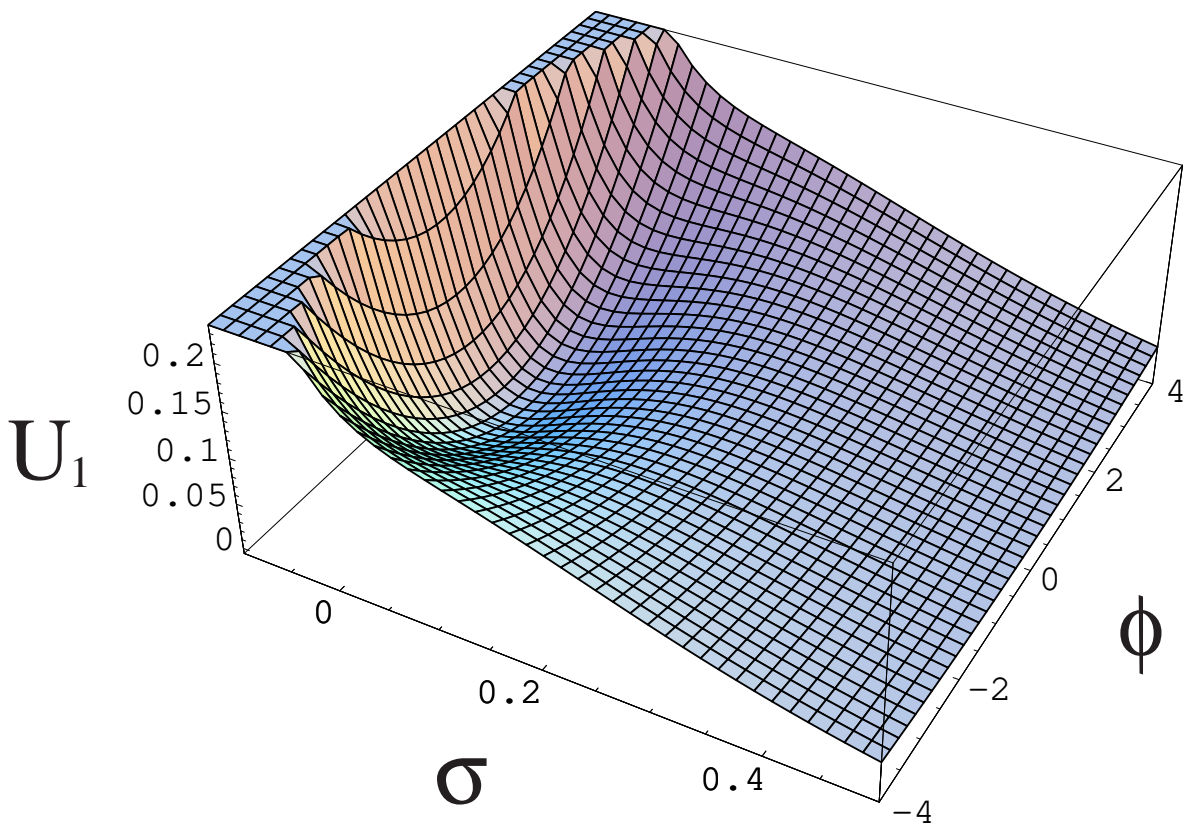

FIG. 1: The effective potential $U_{1}(\sigma)$ which is obtained by introducing the Casimir energy in the sphere compactification with $d=6$. We find that the potential barrier disappears for large values of inflaton. In order to evolve toward the present vacuum state $\phi=\sigma=0$, inflaton is required to be smaller than some critical value $\phi_{c}$. 
Let us consider the dynamics of inflation in the presence of the $\sigma$ field. In the flat FRW background: $d s^{2}=$ $-d t^{2}+a^{2}(t) d \mathbf{x}^{2}$, we find that the homogeneous parts of scalar fields and the scale factor satisfy the following equations of motion by the action (2.8):

$$
\begin{gathered}
\ddot{\phi}+3 H \dot{\phi}+e^{-d \sigma / \sigma_{*}} V^{\prime}(\phi)=0, \\
\ddot{\sigma}+3 H \dot{\sigma}+U^{\prime}(\sigma)-\frac{d}{\sigma_{*}} e^{-d \sigma / \sigma_{*}} V(\phi)=0, \\
H^{2} \equiv\left(\frac{\dot{a}}{a}\right)^{2}=\frac{\kappa^{2}}{3}\left[\frac{1}{2} \dot{\phi}^{2}+e^{-d \sigma / \sigma_{*}} V(\phi)+\frac{1}{2} \dot{\sigma}^{2}+U(\sigma)\right],
\end{gathered}
$$

where $H$ is the Hubble expansion rate.

Fig. 2

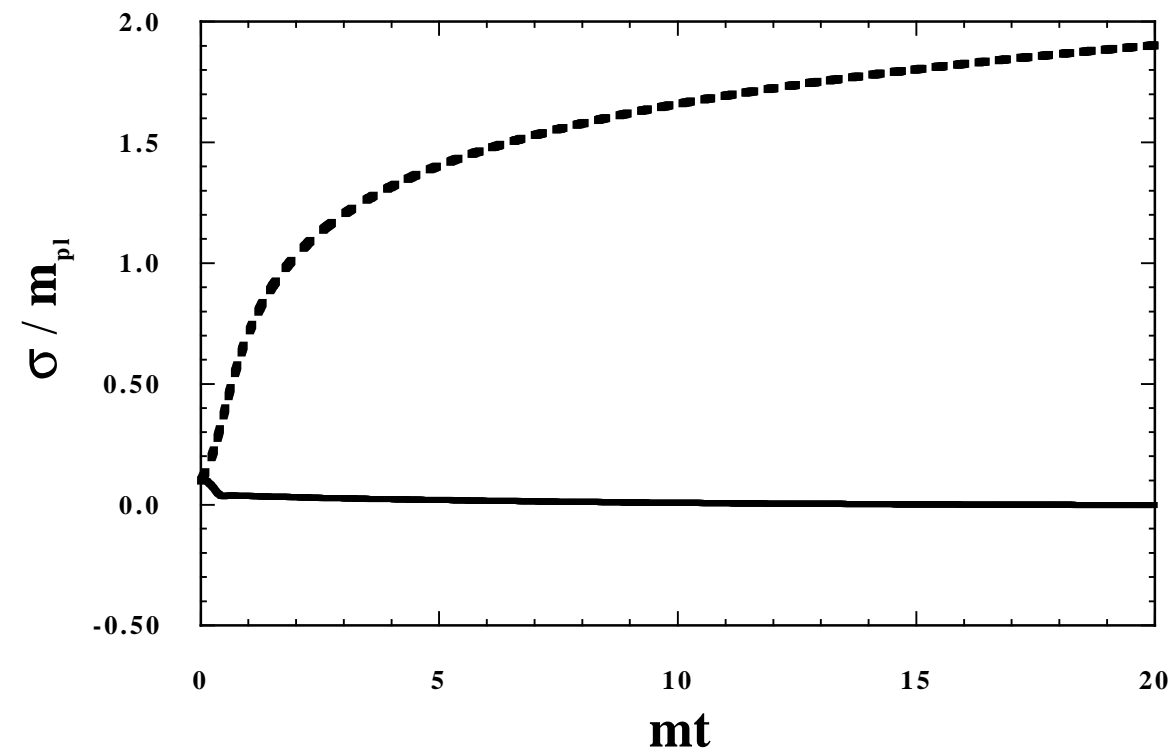

FIG. 2: The evolution of the $\sigma$ field with the initial value of $\sigma_{I}=0.1 m_{\mathrm{pl}}$ for two cases of $\phi_{I}=3 m_{\mathrm{pl}}$ (solid) and $\phi_{I}=4 m_{\mathrm{pl}}$ (dotted) with $d=6$ and $b_{*}=1.0 \times 10^{5} / \mathrm{m}_{\mathrm{pl}}$. When $\phi_{I}=3 m_{\mathrm{pl}}$, the $\sigma$ field evolves toward the potential minimum at $\phi=\sigma=0$ due to the existence of the potential barrier. On the other hand, when $\phi_{I}=4 m_{\mathrm{pl}}$, the internal space grows without limit.

When the initial value of inflaton is larger than $\phi_{c}$, the last term in the l.h.s. of Eq. (2.19) dominates over the third term and the $\sigma$ field evolves toward $\sigma=\infty$ (see Fig. 2). This is the situation we want to avoid. For the values of $\phi<\phi_{c}$, there exists a local minimum at $\sigma=\sigma_{1}$ and a local maximum at $\sigma=\sigma_{2}$ with $\sigma_{2}>\sigma_{1}>0$. As long as the initial value of $\sigma$ exists in the range of $\sigma<\sigma_{2}$, the $\sigma$ field evolves toward the potential minimum at $\sigma=\sigma_{1}$. The value of $\sigma_{1}$ decreases to zero as the $\phi$ field moves toward the potential minimum at $\phi=0$. For the initial values of $\sigma$ and $\phi$ which are finally trapped in the potential minimum at $\sigma=\phi=0$, one may consider that the dynamics of inflation is altered in the presence of the $\sigma$ field. In this case, however, we can numerically confirm that the third term in Eq. (2.19) rapidly makes the $\sigma$ field shift toward the local minimum at $\sigma=\sigma_{1}$ for the values of $b_{*}$ which satisfy the 
condition of Eq. (2.16). Then $\sigma$ begins to roll down along the valley of $\sigma=\sigma_{1}$, and decreases as inflaton approaches its potential minimum. This behavior is found in Fig. 2. The dynamics of inflation is hardly affected by the presence of the $\sigma$ field, and the system can be effectively described by one scalar field $\phi$.

In Fig. 3, we plot the evolution of both the inflaton field and the number of e-foldings $N \equiv \ln \left(a / a_{I}\right)$ during inflation. After the rapid decrease of $\sigma$ at the initial stage, inflaton slowly rolls down along its potential in the usual manner, which results in sufficient inflation to solve several cosmological puzzles. We also find that the number of e-foldings exceeds $N \sim 60$ for the initial value of $\phi_{I}=3 m_{\mathrm{pl}}$. The inflationary period ends when inflaton decreases to $\phi \approx 0.2 m_{\mathrm{pl}}$, which corresponds to the time $m t \approx 20$ in Fig. 3 . In the next section, we investigate the dynamics of field and metric perturbations during preheating.

Fig. 3

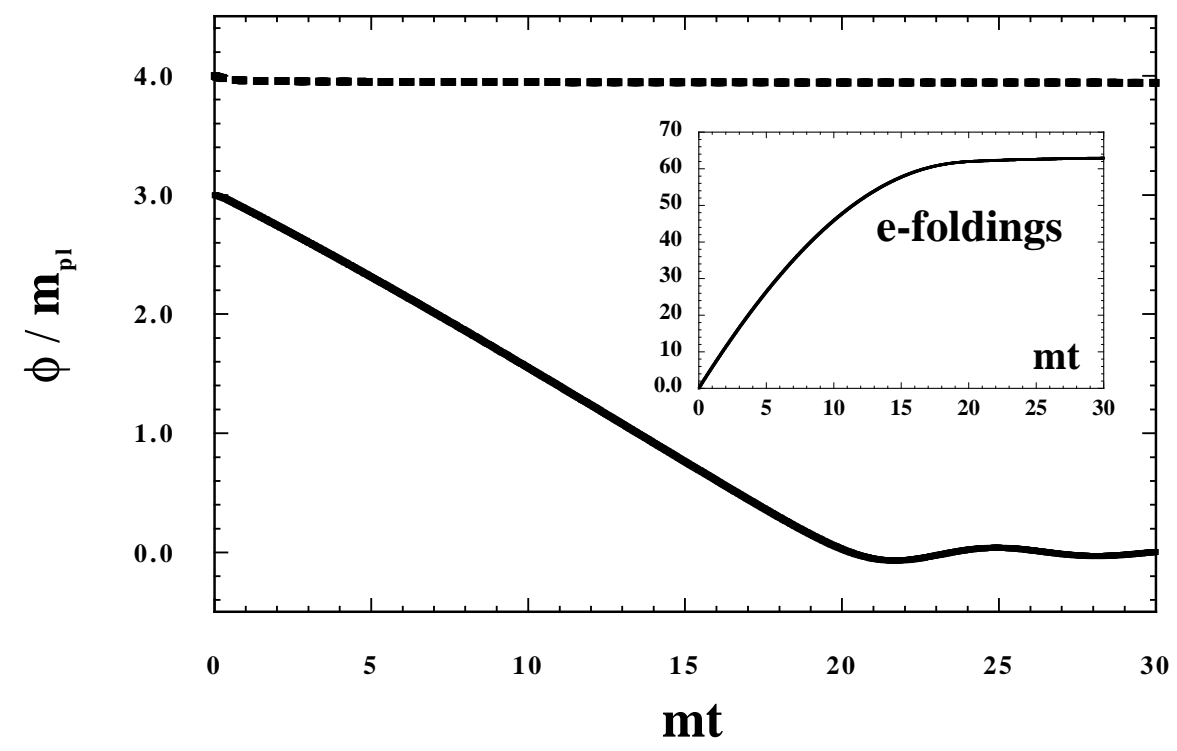

FIG. 3: The evolution of the $\phi$ field during inflation with the initial value of $\sigma_{I}=$ $0.1 m_{\mathrm{pl}}$ for two cases of $\phi_{I}=3 m_{\mathrm{pl}}$ (solid) and $\phi_{I}=4 m_{\mathrm{pl}}$ (dotted) with $d=6$ and $b_{*}=1.0 \times 10^{5} / m_{\mathrm{pl}}$. When $\phi_{I}=3 m_{\mathrm{pl}}$, inflation proceeds in the usual manner while inflaton slowly evolves toward $\phi=0$. When $\phi_{I}=4 m_{\mathrm{pl}}$, the system evolves toward larger values of $\sigma$, and the value of inflaton hardly changes. Inset: Evolution of the number of e-foldings $N$ during inflation for $\phi_{I}=3 m_{\mathrm{pl}}$. We find that $N>60$ is achieved.

\section{PREHEATING WITH EXTRA DIMENSIONS}

After inflation, the system enters the preheating stage during which fluctuations of scalar fields will grow by parametric resonance. We introduce another massless scalar field $\chi$ coupled to inflaton, and adopt the following modified potential instead of (2.13):

$$
V(\phi, \chi)=\frac{1}{2} m^{2} \phi^{2}+\frac{1}{2} g^{2} \phi^{2} \chi^{2}+\tilde{g}^{2} \phi^{3} \chi
$$


Note that we include the interaction term $\tilde{g}^{2} \phi^{3} \chi$ which often appears in supergravity models [31] in addition to the standard term $\frac{1}{2} g^{2} \phi^{2} \chi^{2}$. This provides a way to escape from an inflationary suppression of the $\chi$ field as we will show later.

When we consider fluctuations of scalar fields, metric perturbations should be also taken into account for a consistent study of preheating [32.33]. In fact, inclusion of metric perturbations can change the evolution of field fluctuations significantly in broad classes of models [34 37]. In this paper, we adopt the perturbed metric in the longitudinal gauge in the flat FRW background:

$$
d s^{2}=-(1+2 \Phi) d t^{2}+a^{2}(t)(1-2 \Psi) \delta_{i j} d x^{i} d x^{j}
$$

where $\Phi$ and $\Psi$ are gauge-invariant potentials [38].

Decomposing scalar fields into homogeneous and gauge-invariant fluctuational parts as $\phi(t, \mathbf{x}) \rightarrow \phi(t)+\delta \phi(t, \mathbf{x})$, $\sigma(t, \mathbf{x}) \rightarrow \sigma(t)+\delta \sigma(t, \mathbf{x}), \chi(t, \mathbf{x}) \rightarrow \chi(t)+\delta \chi(t, \mathbf{x})$, and expanding scalar field fluctuations and metric fluctuations by Fourier modes, we obtain the following perturbed equations (see e.g., 33]):

$$
\begin{gathered}
\Phi_{k}=\Psi_{k}, \\
\dot{\Phi}_{k}+H \Phi_{k}=\frac{\kappa^{2}}{2}\left(\dot{\phi} \delta \phi_{k}+\dot{\sigma} \delta \sigma_{k}+\dot{\chi} \delta \chi_{k}\right), \\
3 H \dot{\Phi}_{k}+\left[\frac{k^{2}}{a^{2}}+3 H^{2}-\frac{\kappa^{2}}{2}\left(\dot{\phi}^{2}+\dot{\sigma}^{2}+\dot{\chi}^{2}\right)\right] \Phi_{k} \\
=-\frac{\kappa^{2}}{2}\left(\dot{\phi} \delta \dot{\phi}_{k}+U_{1, \phi} \delta \phi_{k}+\dot{\sigma} \delta \dot{\sigma}_{k}+U_{1, \sigma} \delta \sigma_{k}+\dot{\chi} \delta \dot{\chi}_{k}+U_{1, \chi} \delta \chi_{k}\right), \\
\delta \ddot{\phi}_{k}+3 H \delta \dot{\phi}_{k}+\left[\frac{k^{2}}{a^{2}}+e^{-d \sigma / \sigma_{*}}\left(m^{2}+g^{2} \chi^{2}+6 \tilde{g}^{2} \phi \chi\right)\right] \delta \phi_{k}=4 \dot{\phi} \dot{\Phi}_{k}+2(\ddot{\phi}+3 H \dot{\phi}) \Phi_{k}-U_{1, \sigma \phi} \delta \sigma_{k}-U_{1, \chi \phi} \delta \chi_{k}, \\
\delta \ddot{\sigma}_{k}+3 H \delta \dot{\sigma}_{k}+\left[\frac{k^{2}}{a^{2}}+U^{\prime \prime}(\sigma)+\frac{d^{2}}{\sigma_{*}^{2}} e^{-d \sigma / \sigma_{*}}\left(\frac{1}{2} m^{2} \phi^{2}+\frac{1}{2} g^{2} \phi^{2} \chi^{2}+\tilde{g}^{2} \phi^{3} \chi\right)\right] \delta \sigma_{k} \\
=4 \dot{\sigma} \dot{\Phi}_{k}+2(\ddot{\sigma}+3 H \dot{\sigma}) \Phi_{k}-U_{1, \phi \sigma} \delta \phi_{k}-U_{1, \chi \sigma} \delta \chi_{k}, \\
\delta \ddot{\chi}_{k}+3 H \delta \dot{\chi}_{k}+\left(\frac{k^{2}}{a^{2}}+g^{2} \phi^{2} e^{-d \sigma / \sigma_{*}}\right) \delta \chi_{k}=4 \dot{\chi} \dot{\Phi}_{k}+2(\ddot{\chi}+3 H \dot{\chi}) \Phi_{k}-U_{1, \sigma \chi} \delta \sigma_{k}-U_{1, \phi \chi} \delta \phi_{k},
\end{gathered}
$$

where $U_{1, \phi \sigma}, U_{1, \chi \sigma}$, and $U_{1, \chi \phi}$ are expressed as $U_{1, \phi \sigma}=-d e^{-d \sigma / \sigma_{*}}\left(m^{2}+g^{2} \chi^{2}+3 \tilde{g}^{2} \phi \chi\right) \phi / \sigma_{*}, U_{1, \chi \sigma}=-d e^{-d \sigma / \sigma_{*}}\left(g^{2} \chi+\right.$ $\left.\tilde{g}^{2} \phi\right) \phi^{2} / \sigma_{*}$, and $U_{1, \chi \phi}=e^{-d \sigma / \sigma_{*}}\left(2 g^{2} \phi \chi+3 \tilde{g}^{2} \phi^{2}\right)$, respectively.

The relation (3.3) indicates that the anisotropic stress vanishes at linear order. Eliminating the $\dot{\Phi}_{k}$ term in Eqs. (3.4) and (3.5), we find

$$
\left(\frac{k^{2}}{a^{2}}-\frac{\kappa^{2}}{2} \sum_{J} \dot{\varphi}_{J}^{2}\right) \Phi_{k}=-\frac{\kappa^{2}}{2} \sum_{J}\left(\dot{\varphi}_{J} \delta \dot{\varphi}_{J k}+3 H \dot{\varphi}_{J} \delta \varphi_{J k}+U_{1, \varphi_{J}} \delta \varphi_{J k}\right),
$$

where $\varphi_{J}(J=1,2,3)$ correspond to the scalar fields $\phi, \sigma, \chi$, respectively. Eq. (3.9) shows that metric perturbations are known when evolutions of scalar fields are determined. When field fluctuations are amplified, it is expected that 
this stimulates the growth of metric perturbations by Eq. (3.9). The enhancement of metric perturbations will also assist the excitation of field perturbations as is found by Eqs. (3.6)-(3.8).

Parametric amplification of field fluctuations affects evolutions of the background quantities. Since the $\chi$ fluctuation generally grows faster than other field fluctuations, we include this contribution in the background equations as

$$
\begin{gathered}
\ddot{\phi}+3 H \dot{\phi}+e^{-d \sigma / \sigma_{*}}\left(m^{2}+g^{2}\left\langle\chi^{2}\right\rangle+3 \tilde{g}^{2} \phi \chi\right) \phi=0, \\
\ddot{\sigma}+3 H \dot{\sigma}+U^{\prime}(\sigma)-\frac{d}{\sigma_{*}} e^{-d \sigma / \sigma_{*}}\left(\frac{1}{2} m^{2} \phi^{2}+\frac{1}{2} g^{2} \phi^{2}\left\langle\chi^{2}\right\rangle+\tilde{g}^{2} \phi^{3} \chi\right)=0, \\
\ddot{\chi}+3 H \dot{\chi}+e^{-d \sigma / \sigma_{*}}\left(g^{2} \phi^{2} \chi+\tilde{g}^{2} \phi^{3}\right)=0, \\
H^{2}=\frac{\kappa^{2}}{3}\left[\frac{1}{2} \dot{\sigma}^{2}+U(\sigma)+\frac{1}{2} \dot{\phi}^{2}+\frac{1}{2} \dot{\chi}^{2}+e^{-d \sigma / \sigma_{*}}\left(\frac{1}{2} m^{2} \phi^{2}+\frac{1}{2} g^{2} \phi^{2}\left\langle\chi^{2}\right\rangle+\tilde{g}^{2} \phi^{3} \chi\right)\right],
\end{gathered}
$$

where the spatial average of the $\chi$ fluctuation is defined by

$$
\left\langle\chi^{2}\right\rangle=\frac{1}{2 \pi^{2}} \int k^{2}\left|\delta \chi_{k}\right|^{2} d k
$$

Let us examine evolutions of the background fields and the scale factor. As is found in the previous section, the $\sigma$ field rapidly decreases during inflation compared with the $\phi$ field, and the condition $\sigma \ll \sigma_{*}=\sqrt{d(d+2) / 16 \pi} m_{\mathrm{pl}}$ holds at the beginning of preheating. Then, in the stage where the $\chi$ fluctuation is not significantly enhanced, Eqs. (3.10)-(3.13) can be approximately written as

$$
\begin{gathered}
\ddot{\phi}+3 H \dot{\phi}+m^{2} \phi=0, \\
\ddot{\sigma}+3 H \dot{\sigma}+\frac{2(d-1)}{b_{*}^{2}} \sigma=0, \\
\ddot{\chi}+3 H \dot{\chi}+g^{2} \phi^{2} \chi+\tilde{g}^{2} \phi^{3}=0, \\
H^{2}=\frac{\kappa^{2}}{3}\left(\frac{1}{2} \dot{\phi}^{2}+\frac{1}{2} m^{2} \phi^{2}\right) .
\end{gathered}
$$

Making use of the time-averaged relation $\left\langle\frac{1}{2} \dot{\phi}^{2}\right\rangle_{T}=\left\langle\frac{1}{2} m^{2} \phi^{2}\right\rangle_{T}$ during the oscillating stage of inflaton, the evolution of inflaton is analytically expressed by Eqs. (3.15) and (3.18) as

$$
\phi=\Phi(t) \sin m t, \quad \text { with } \quad \Phi(t)=\frac{m_{\mathrm{pl}}}{\sqrt{3 \pi} m t} .
$$

The coherent oscillation of inflaton begins when $\Phi\left(t_{i}\right) \sim 0.2 m_{\mathrm{pl}}$, and we set the initial time as $m t=\pi / 2$ as in Ref. [16]. The scale factor evolves as $a \sim t^{2 / 3}$ since the system is dominated by the oscillation of the massive inflaton field. Although the $\sigma$ field oscillates with a frequency $\sqrt{2(d-1)} / b_{*}$, its amplitude is very small relative to that of the $\phi$ field. For example, in the simulation of Fig. 2, the amplitude of $\sigma$ at the start of preheating is found to be about $10^{-5} m_{\mathrm{pl}}$. 
If we neglect metric perturbations, the evolution of the $\delta \chi_{k}$ fluctuation can be studied analytically at the linear stage of preheating. Ignoring the r.h.s. of Eq. (3.8) and introducing a new scalar field $\delta X_{k}=a^{3 / 2} \delta \chi_{k}$, Eq. (3.8) reads

$$
\frac{d^{2}}{d t^{2}} \delta X_{k}+\left[\frac{k^{2}}{a^{2}}+g^{2} \phi^{2}-\frac{3}{4}\left(\frac{2 \ddot{a}}{a}+\frac{\dot{a}^{2}}{a^{2}}\right)\right] \delta X_{k}=0 .
$$

The last term in Eq. (3.20) which corresponds to the pressure term can be neglected during the oscillating stage of inflaton. Then Eq. (3.20) is reduced to the well-known Mathieu equation [39],

$$
\frac{d^{2}}{d z^{2}} \delta X_{k}+\left(A_{k}-2 q \cos 2 z\right) \delta X_{k}=0
$$

where $z=m t$ and

$$
A_{k}=2 q+\frac{k^{2}}{(m a)^{2}}, \quad q=\frac{g^{2} \Phi^{2}(t)}{4 m^{2}} .
$$

Then the value of $q$ at the beginning of preheating is estimated as

$$
q_{i} \approx 10^{10} \times g^{2},
$$

where we used $\Phi\left(t_{i}\right) \approx 0.2 m_{\mathrm{pl}}$ and $m \sim 10^{-6} m_{\mathrm{pl}}$. The coupling $g \lesssim 10^{-5}$ yields $q_{i} \lesssim 1$, which is generally called the narrow resonance. In this case, parametric resonance is weak in an expanding universe. However, when $q_{i} \gg 1$, it was pointed out in Ref. [15] that the $\chi$ particle production can be efficient in spite of the decrease of $q$ due to cosmic expansion, which was later confirmed by numerical calculations in Ref. [18]. In this case, the $\delta \chi_{k}$ field initially lies in the broad resonance regime as long as $k$ is not so large relative to $m a$, and it jumps over many stability and instability bands with the decrease of $q$. This was termed stochastic resonance in Ref. [16], in which the $\delta \chi_{k}$ fluctuation increases stochastically overcoming the diluting effect by the expansion of the universe. For $g \gtrsim 3 \times 10^{-4}$, the backreaction effect of created $\chi$ particles becomes important, which results in the termination of parametric resonance. In this case, since the coherent oscillation of the inflaton field is broken by the growth of the $\delta \chi_{k}$ fluctuation, the analytical method based on the Mathieu equation is no longer applied. In this respect, several numerical works have been done by making use of mean field approximations or fully nonlinear calculations. In the Hartree approximation the final variance of the $\chi$ field is estimated as $\left\langle\chi^{2}\right\rangle_{f} \propto q^{-1 / 2}$ [18], while in the fully nonlinear calculations it was found to be $\left\langle\chi^{2}\right\rangle_{f} \propto q^{-1}$ for the case of $q \gg 1$ [22].

As for the $\delta \sigma_{k}$ field, there exist resonance terms in the 1.h.s. of Eq. (3.7) which may lead to the enhancement of the fluctuation of dilaton. If we neglect the effect of metric perturbations in the r.h.s. of Eq. (3.7) and making use of the relation $|\sigma| \ll \sigma_{*}$ during preheating, the equation of the $\delta \sigma_{k}$ field can be approximately written as

$$
\delta \ddot{\sigma}_{k}+3 H \delta \dot{\sigma}_{k}+\left[\frac{k^{2}}{a^{2}}+\frac{2(d-1)}{b_{*}^{2}}+\frac{8 \pi d}{d+2}\left(\frac{m}{m_{\mathrm{pl}}}\right)^{2} \phi^{2}\right] \delta \sigma_{k}=0 .
$$

Defining a new scalar field $\delta \Sigma_{k}=a^{3 / 2} \delta \sigma_{k}$ and ignoring the contribution from the pressure term, we obtain

$$
\frac{d^{2}}{d z^{2}} \delta \Sigma_{k}+\left(A_{k}-2 q \cos 2 z\right) \delta \Sigma_{k}=0,
$$

where 


$$
A_{k}=2 q+\frac{2(d-1)}{b_{*}^{2} m^{2}}+\frac{k^{2}}{(m a)^{2}}, \quad q=\frac{2 d}{3(d+2) z^{2}} .
$$

For $d \geq 1, q_{i} \lesssim 1$ at the beginning of preheating. Moreover, since $q$ decreases as $q \sim t^{-2}$, parametric resonance is very weak. Namely, in the unperturbed metric, analytic estimates indicate that the dilaton fluctuation does not grow during preheating. We also find from Eq. (3.6) that the enhancement of the inflaton fluctuation can not be expected in the absence of metric perturbations.

Let us proceed to the case where metric perturbations are taken into account. From Eq. (3.4), we can expect that the growth of the $\delta \chi_{k}$ field will enhance metric perturbations. On the other hand, it was pointed out in Refs. 40 43] that the amplitude of super-Hubble fluctuations in the $\delta \chi_{k}$ field is severely damped during inflation in the case where $g \phi$ is much larger than the Hubble expansion rate $H$ with a model of $V(\phi, \chi)=\frac{1}{2} m^{2} \phi^{2}+\frac{1}{2} g^{2} \phi^{2} \chi^{2}$. Later, Bassett et al. [35] showed that inclusion of the interaction $\tilde{g}^{2} \phi^{3} \chi$ protects super-Hubble $\delta \chi_{k}$ fluctuations from being suppressed. In what follows, we will consider both cases of $\tilde{g}=0$ and $\tilde{g} \neq 0$ separately.

\section{A. Case of $\tilde{g}=0$}

Let us first estimate the amplitude of super-Hubble $\delta \chi_{k}$ modes at the beginning of preheating. When $\tilde{g}=0$, the adiabatic solution for $\delta \chi_{k}$ during inflation is expressed as

$$
\delta \chi_{k}=\frac{a^{-3 / 2}}{\sqrt{2 \omega_{k}}} e^{-i \omega_{k} t}
$$

where $\omega_{k}^{2}=k^{2} / a^{2}+g^{2} \phi^{2} e^{-d \sigma / \sigma_{*}} \approx k^{2} / a^{2}+g^{2} \phi^{2}$. In order to lead to efficient $\chi$ particle production, the resonance parameter is required to be $q=g^{2} \phi^{2} / 4 m^{2} \gg 1$. In this case, the effective mass of the $\delta \chi_{k}$ field is much larger than the Hubble expansion rate $H \sim m$ during inflation. Then the amplitude of the super-Hubble $\delta \chi_{k}$ field for modes relevant for structure formation is estimated as $\left|\delta \chi_{k}\right| \sim a^{-3 / 2} / \sqrt{g \phi}$, which exponentially decreases during inflation. On the other hand, since the effective mass of the $\delta \phi_{k}$ field in the l.h.s. of Eq. (3.6) is comparable to the Hubble rate $H$, the super-Hubble inflaton fluctuation is not affected by the suppression during inflation.

As for the $\delta \sigma_{k}$ field, its effective mass for super-Hubble modes is given by Eq (3.24) as

$$
m_{\mathrm{eff}}^{2}=\left[\frac{2(d-1)}{b_{*}^{2} m^{2}}+\frac{8 \pi d}{d+2}\left(\frac{\phi}{m_{\mathrm{pl}}}\right)^{2}\right] m^{2} .
$$

For the typical scale $b_{*}$ which is determined by Eq. (2.16) and the initial value of inflaton $\phi_{I} \gtrsim 3 m_{\mathrm{pl}}, m_{\mathrm{eff}}$ is estimated as $m_{\text {eff }}^{2} \gtrsim 100 m^{2} \sim 10 H^{2}$. Hence the super-Hubble $\delta \sigma_{k}$ fluctuation will be also affected by the inflationary suppression, which is relevant for small $b_{*}$ and large initial values of $\phi$. We can roughly estimate the amplitude of super-Hubble $\delta \sigma_{k}$ modes by Eq. (3.7) during inflation. Neglecting the contributions of the $\chi$ field and the time derivative terms of $\sigma$ and $\delta \sigma_{k}$, we obtain the amplitude of $\delta \sigma_{k}$ at the start of preheating as

$$
\left|\delta \sigma_{k}\left(t_{i}\right)\right| \approx \frac{2}{(d-1)} \sqrt{\frac{\pi d}{d+2}} \frac{\phi}{m_{\mathrm{pl}}}\left(b_{*} m\right)^{2}\left|\delta \phi_{k}\left(t_{i}\right)\right| .
$$

For example, when $d=6$, since $b_{*}$ is constrained as $b_{*} \lesssim 1.1 \times 10^{5} / m_{\mathrm{pl}}$, Eq. (3.29) yields

$$
\left|\delta \sigma_{k}\left(t_{i}\right)\right| \lesssim 10^{-3}\left|\delta \phi_{k}\left(t_{i}\right)\right|
$$


This means that the suppression effect of super-Hubble modes is weak compared with the $\delta \chi_{k}$ field as long as $b_{*}$ is not much smaller than its upper bound.

Let us estimate the impact on metric perturbations by the growth of field fluctuations. First, we introduce the power spectrum of $\Phi_{k}$ :

$$
\mathcal{P}(k)=\frac{k^{3}}{2 \pi^{2}}\left|\Phi_{k}\right|^{2}=\frac{\left|\tilde{\Phi}_{k}\right|^{2}}{2 \pi^{2}}
$$

where $\tilde{\Phi}_{k} \equiv k^{3 / 2} \Phi_{k}$. Defining new scalar fields $\tilde{\varphi}_{J} \equiv \varphi_{J} / m_{\mathrm{pl}}$ and $\delta \tilde{\varphi}_{J k} \equiv k^{3 / 2} \delta \varphi_{J k} / m_{\mathrm{pl}}(J=1,2,3)$, we obtain the following relation from Eq. (3.4):

$$
\dot{\tilde{\Phi}}_{k}+H \tilde{\Phi}_{k}=4 \pi\left(\dot{\tilde{\phi}} \delta \tilde{\phi}_{k}+\dot{\tilde{\sigma}} \delta \tilde{\sigma}_{k}+\dot{\tilde{\chi}} \delta \tilde{\chi}_{k}\right)
$$

For super-Hubble modes $k \ll a H$, the amplitude of $\delta \tilde{\chi}_{k}$ is written as $\left|\delta \tilde{\chi}_{k}\right| \approx \bar{k}^{3 / 2}\left(m / m_{\mathrm{pl}}\right) \sqrt{m /(2 g \phi)}$ where $\bar{k} \equiv$ $k /\left(m a_{i}\right)$ with $a_{i}$ the scale factor at the onset of preheating. Since the cosmological modes correspond to $\bar{k} \sim e^{-60} \sim$ $10^{-26},\left|\delta \tilde{\chi}_{k}\right|$ is estimated as $\left|\delta \tilde{\chi}_{k}\right| \lesssim 10^{-45}$ for the broad resonance case $q \gg 1$. The homogeneous part of the $\chi$ field is also affected by this strong suppression [see Eq. (3.12) with $\tilde{g}=0$ ]. At the beginning of preheating, the $\dot{\chi} \delta \chi_{k}$ term in the r.h.s. of Eq. (3.4) is very small relative to the $\dot{\phi} \delta \phi_{k}$ term. Although super-Hubble $\delta \chi_{k}$ fluctuations exhibit parametric amplification during preheating, it increases only by the factors $10^{4}-10^{5}$ for the coupling of $g=3 \times 10^{-4}-10^{-2} 41$. Hence we can expect that the excitement of the $\delta \chi_{k}$ fluctuation hardly affects the evolution of super-Hubble metric perturbations by analytic estimates.

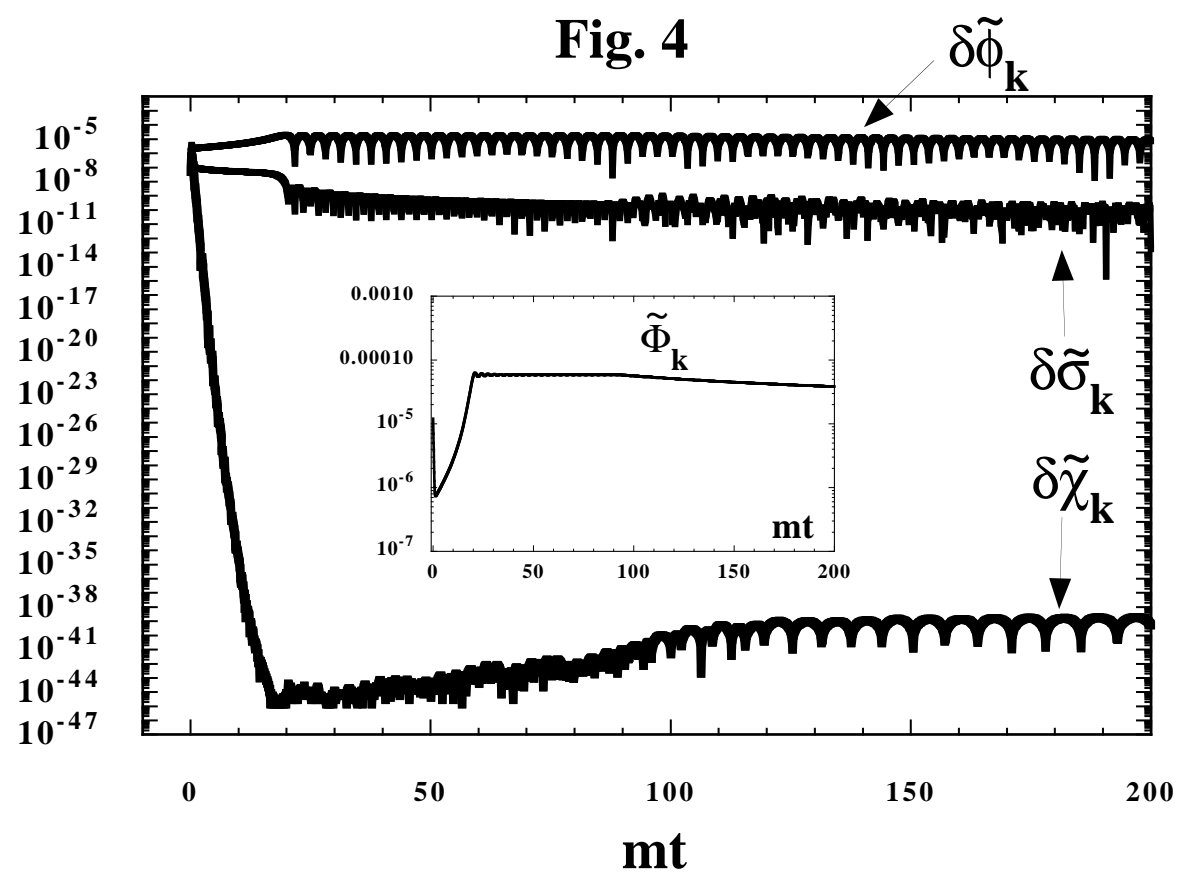

FIG. 4: The evolutions of field perturbations $\delta \tilde{\phi}_{k}, \delta \tilde{\sigma}_{k}, \delta \tilde{\chi}_{k}$ for a super-Hubble mode $\bar{k}=10^{-26}$ with $g=1.0 \times 10^{-3}, d=6, b_{*}=1.0 \times 10^{5} / m_{\mathrm{pl}}$, and $m=10^{-6} m_{\mathrm{pl}}$. See the text for the initial conditions of scalar fields. Note that we start integrating about 60 e-foldings before the beginning of preheating. Inset: The evolution of the metric perturbation $\tilde{\Phi}_{k}$ for a super-Hubble mode $\bar{k}=10^{-26}$. 
We are also concerned with whether super-Hubble $\delta \sigma_{k}$ fluctuations are enhanced or not during preheating. Although the inflationary suppression for $\delta \sigma_{k}$ is not so significant as compared with the $\delta \chi_{k}$ case, we have to keep in mind that $\delta \sigma_{k}$ can not be enhanced unless metric perturbations are taken into account. In order to amplify super-Hubble metric perturbations, we generally require some scalar fields such as $\chi$ which exhibit parametric amplification even in the absence of metric perturbations. However, when $\tilde{g}=0$, the $\delta \chi_{k}$ fluctuation in large scales is strongly suppressed. It is expected that the super-Hubble fluctuation of dilaton will be held static during preheating, because the $\sigma$ field will not play a dominant role to stimulate the enhancement of metric perturbations.

In order to verify the above estimates, we numerically solved perturbed equations (3.3)-(3.8) along with background equations (3.10)-(3.13). In Fig. 4, we plot the evolutions of field perturbations $\delta \tilde{\phi}_{k}, \delta \tilde{\sigma}_{k}, \delta \tilde{\chi}_{k}$, and the metric perturbation $\tilde{\Phi}_{k}$ during inflation and preheating for a super-Hubble mode $\bar{k}=10^{-26}$ with $g=1.0 \times 10^{-3}, d=6$, $b_{*}=1.0 \times 10^{5} / m_{\mathrm{pl}}$, and $m=10^{-6} \mathrm{~m}_{\mathrm{pl}}$. The initial values of homogeneous scalar fields are chosen as $\phi_{I}=3.0 m_{\mathrm{pl}}$, $\sigma_{I}=0.1 m_{\mathrm{pl}}$, and $\chi_{I}=1.0 \times 10^{-3} m_{\mathrm{pl}}$. As for the initial field perturbations, we take

$$
\left|\delta \varphi_{J k}\right|=\frac{1}{\sqrt{2 \omega_{J k}}}, \quad\left|\delta \dot{\varphi}_{J k}\right|=\omega_{J k}\left|\delta \varphi_{J k}\right|
$$

where $\omega_{J k}^{2} \equiv k^{2} / a^{2}+m_{\varphi_{J}}^{2}(J=1,2,3)$ with $m_{\varphi_{J}}$ is the effective mass of the each scalar field in the l.h.s. of Eqs. (3.6)-(3.8).

In Fig. 4 , the $\delta \chi_{k}$ fluctuation is exponentially suppressed during inflation $(0<m t \lesssim 20)$, yielding $\delta \tilde{\chi}_{k} \sim 10^{-45}$ at the beginning of preheating. Although $\delta \chi_{k}$ is enhanced by parametric resonance for $m t \gtrsim 20$, the final value is very small as $\delta \tilde{\chi}_{k} \sim 10^{-40}$. The amplitude of $\delta \sigma_{k}$ is by three orders of magnitude smaller than that of $\delta \phi_{k}$ as is analytically estimated by Eq. (3.29) at the end of inflation. We also find in Fig. 4 that both of super-Hubble $\delta \sigma_{k}$ and $\delta \phi_{k}$ fluctuations do not grow during preheating, which means that field fluctuations are not assisted by the presence of metric perturbations. As is found in the inset of Fig. 4, super-Hubble metric perturbations remain almost constant during preheating. In the case of $\tilde{g}=0$, the usual prediction of the inflationary spectrum in large scales is not likely to be modified, and the analysis neglecting metric perturbations gives almost the same results as compared with the perturbed metric case. As a result, the fluctuation of dilaton on super-Hubble scales can not be amplified during preheating.

Let us next consider smaller scales which are within the Hubble radius at the beginning of preheating. Since this corresponds to the modes $k \gtrsim a_{i} H_{i}$, the condition $k^{2} / a^{2}>g^{2} \phi^{2}$ holds in most stage of inflation. Hence the amplitude of these modes during inflation is approximately expressed by Eq. (3.27) as

$$
\left|\delta \chi_{k}\right| \approx \frac{1}{a \sqrt{2 k}} .
$$

The r.h.s. of Eq. (3.34) decreases slower compared with the super-Hubble modes. In fact, for the modes of $k^{2} / a_{i}^{2} \gtrsim g^{2} \phi_{i}^{2}$ at the start of preheating, we obtain

$$
\left|\delta \tilde{\chi}_{k}\left(t_{i}\right)\right| \approx \frac{1}{\sqrt{2}} \frac{m}{m_{\mathrm{pl}}} \bar{k}
$$

For sub-Hubble modes $\bar{k}=k /\left(m a_{i}\right) \gtrsim 1$, we find $\left|\delta \tilde{\chi}_{k}\left(t_{i}\right)\right| \gtrsim 10^{-6}$, which is much larger than in the super-Hubble case. However, since the homogeneous part of the $\chi$ field is severely damped, the $\dot{\chi} \delta \chi_{k}$ term in the r.h.s. of Eq. (3.4) is still 
much smaller than the $\dot{\phi} \delta \phi_{k}$ term at the beginning of preheating. This indicates that parametric amplification of the $\delta \chi_{k}$ fluctuation will not lead to the growth of sub-Hubble metric perturbations. We have numerically confirmed that metric perturbations and the $\delta \sigma_{k}$ fluctuation for sub-Hubble modes $1 \lesssim k /\left(m a_{i}\right) \lesssim 100$ are not relevantly enhanced for the coupling of $3 \times 10^{-4} \lesssim g \lesssim 10^{-2}$ (see Fig. 5). However, we have to caution that including the second order metric backreaction effect [44] will lift the homogeneous $\chi$ field, which may assist the enhancement of metric and field fluctuations in sub-Hubble modes as was mentioned in Ref. [41. The full backreaction issues are left for the future work.

Fig. 5

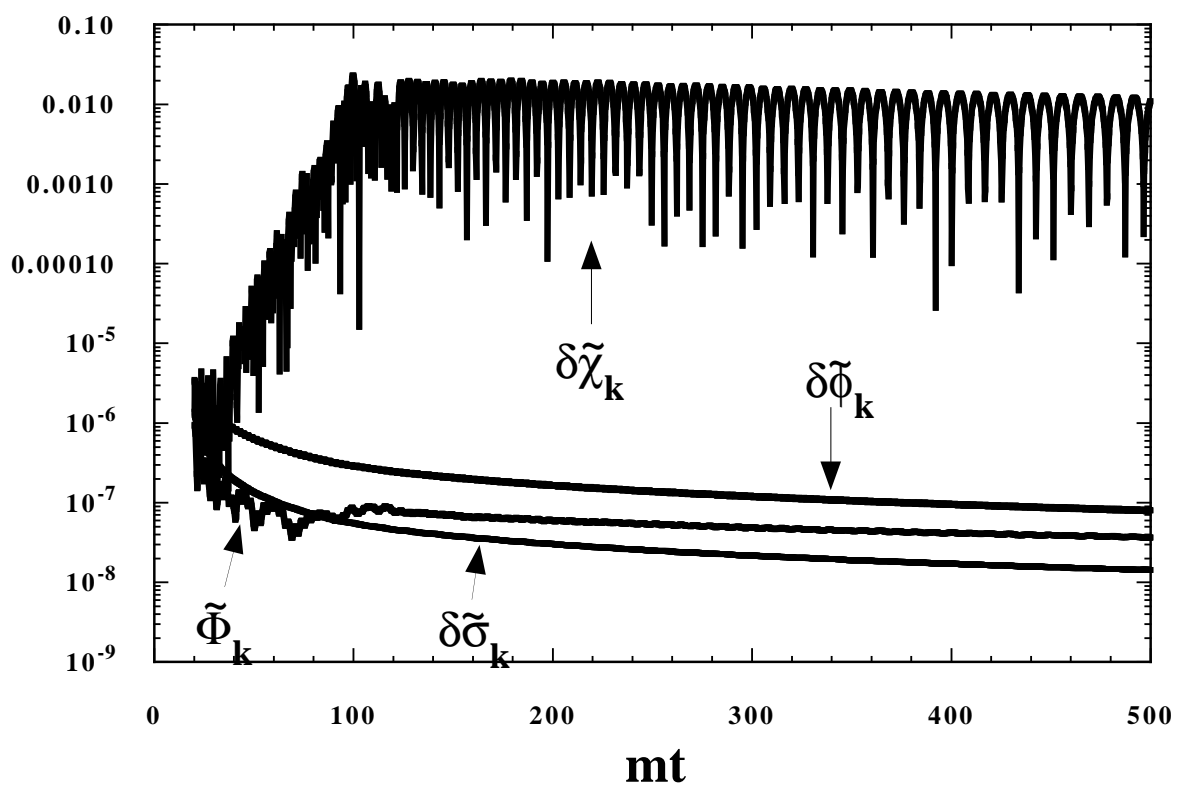

FIG. 5: The evolutions of field perturbations $\delta \tilde{\phi}_{k}, \delta \tilde{\sigma}_{k}, \delta \tilde{\chi}_{k}$, and the metric perturbation $\tilde{\Phi}_{k}$ for a sub-Hubble mode $\bar{k}=5$ with $g=1.0 \times 10^{-3}, d=6, b_{*}=1.0 \times 10^{5} / m_{\mathrm{pl}}$, and $m=10^{-6} m_{\mathrm{pl}}$. Note that we start integrating from the beginning of preheating.

In the case of $\tilde{g}=0$, we argue that the dilaton fluctuation as well as metric perturbations in both super- and sub-Hublle scales can not be strongly amplified during preheating.

\section{B. Case of $\tilde{g} \neq 0$}

If the coupling $\tilde{g}^{2} \phi^{3} \chi$ is taken into account, the suppression of the super-Hubble $\delta \chi_{k}$ fluctuation can be avoided. For super-Hubble modes, neglecting derivative terms of $\delta \chi_{k}$ and $\chi$ fields as well as the $\delta \sigma_{k}$ term in Eq. (3.8), we find the following relation at the end of inflation:

$$
\delta \chi_{k} \approx-3\left(\frac{\tilde{g}}{g}\right)^{2} \delta \phi_{k} .
$$

As for the homogeneous part of the $\chi$ field, Eq. (3.17) implies

$$
\chi \approx-\left(\frac{\tilde{g}}{g}\right)^{2} \phi
$$


We find from Eqs. (3.36) and (3.37) that both of super-Hubble $\delta \chi_{k}$ fluctuations and the homogeneous $\chi$ field are not severely suppressed compared with the $\tilde{g}=0$ case. Then we can expect that the growth of the $\dot{\chi} \delta \chi_{k}$ term in the r.h.s. of Eq. (3.4) during preheating may lead to the enhancement of super-Hubble metric perturbations.

Although larger values of $\tilde{g}$ will surely escape the inflationary suppression, we have to take care that this may prevent the successful inflationary scenario. During inflation, the frequency $\Omega_{\phi}$ of the inflaton condensate is estimated by making use of Eq. (3.37) as

$$
\Omega_{\phi}^{2} \equiv m^{2}+g^{2} \chi^{2}+3 e^{-d \sigma / \sigma_{*}} \tilde{g}^{2} \phi \chi \approx m^{2}\left[1-\frac{2 \tilde{g}^{2} \phi^{2}}{m^{2}}\left(\frac{\tilde{g}}{g}\right)^{2}\right],
$$

where we used the relation $e^{-d \sigma / \sigma_{*}} \approx 1$. Eq. (3.38) indicates that larger values of $\tilde{g}$ make $\Omega_{\phi}^{2}$ negative, and will lead to the unphysical result that $\phi$ increases by negative instability. In order to avoid this, $\Omega_{\phi}^{2}$ should be positive at the start of inflation $\left(\phi_{I} \sim 3 m_{\mathrm{pl}}\right)$. Then the ratio $\tilde{g} / g$ is constrained as

$$
\frac{\tilde{g}}{g} \lesssim \frac{5 \times 10^{-4}}{\sqrt{g}}
$$

where we used $m \sim 10^{-6} m_{\mathrm{pl}}$. For an efficient $\chi$ particle production, the coupling $g$ is required to be $g \gtrsim 3.0 \times 10^{-4}$ [16], which leads to the constraint: $\tilde{g} / g \lesssim 2.9 \times 10^{-2}$. The upper bound of $\tilde{g} / g$ decreases with the increase of $g$ as is found by Eq. (3.39). For example, $\tilde{g} / g \lesssim 1.6 \times 10^{-2}$ for $g=1.0 \times 10^{-3}$, and $\tilde{g} / g \lesssim 5.0 \times 10^{-3}$ for $g=1.0 \times 10^{-2}$. Although larger values of $g$ are favorable for the rapid growth of the $\delta \chi_{k}$ fluctuation, this simultaneously results in the stronger suppression for $\delta \chi_{k}$ and $\chi$ in Eqs. (3.36) and (3.37).

We have numerically examined the dynamics of preheating in the coupling regimes $g=3 \times 10^{-4}-10^{-2}$ with $\tilde{g}$ constrained by Eq. (3.39), and also checked that the inflationary period proceeds in the usual manner. In Fig. 6, the evolutions of field perturbations $\delta \phi_{k}, \delta \chi_{k}, \delta \sigma_{k}$ and the metric perturbation $\Phi_{k}$ are depicted for a super-Hubble mode $\bar{k}=10^{-26}$ with $g=5.0 \times 10^{-4}, \tilde{g} / g=2.0 \times 10^{-2}$. We start integrating about 60 e-foldings before the beginning of preheating, and choose initial conditions $\phi_{I}=3.0 m_{\mathrm{pl}}, \sigma_{I}=0.1 m_{\mathrm{pl}}$, and $\chi_{I}=-(\tilde{g} / g)^{2} \phi_{I}$ for the homogeneous part, and use Eq. (3.33) for the fluctuational parts. In this case, the analytic estimation (3.36) implies the relation $\left|\delta \chi_{k}\left(t_{i}\right)\right| \approx 10^{-3}\left|\delta \phi_{k}\left(t_{i}\right)\right|$ at the onset of preheating $(m t \approx 20)$, which can be easily confirmed in Fig. 6(a). The superHubble $\delta \chi_{k}$ fluctuation starts to grow from $m t \approx 30$ by parametric resonance, and catches up the $\delta \phi_{k}$ fluctuation at $m t \approx 120$. At this stage, the backreaction effect of the produced $\chi$ particle begins to destroy the coherent oscillation of the $\phi$ field [see the inset of Fig. 6(a)]. In spite of this, the amplification of the $\delta \chi_{k}$ fluctuation still takes place before the oscillation of $\phi$ is completely broken at $m t \approx 220$. For $140 \lesssim m t \lesssim 170$, the super-Hubble $\delta \phi_{k}$ fluctuation is enhanced by about two orders of magnitude. This occurs in the perturbed metric case where the r.h.s. of Eq. (3.6) stimulates the excitement of the $\delta \phi_{k}$ fluctuation. However, since the increase of $\delta \chi_{k}$ is weakened by the backreaction effect of created particles, the period during which the $\delta \phi_{k}$ fluctuation is enhanced does not continue long. In Fig. 6(b), we find that the super-Hubble metric perturbation $\Phi_{k}$ begins to oscillate for $m t \gtrsim 180$, which is due to the enhancement of field fluctuations. However, $\Phi_{k}$ does not increase even by one order of magnitude from the beginning of preheating. This is mainly because the backreaction effect restricts the rapid increase of $\delta \chi_{k}$ soon after the super-Hubble $\delta \chi_{k}$ fluctuation catches up $\delta \phi_{k}$. Although one may think that larger values of $\tilde{g}$ will lead to the strong amplification of $\Phi_{k}$, 
Eq. (3.39) constrains the coupling as $\tilde{g} / g \lesssim 2.2 \times 10^{-2}$ with $g=5.0 \times 10^{-4}$, in which case the super-Hubble metric perturbation can not be strongly excited.

Fig. 6(a)

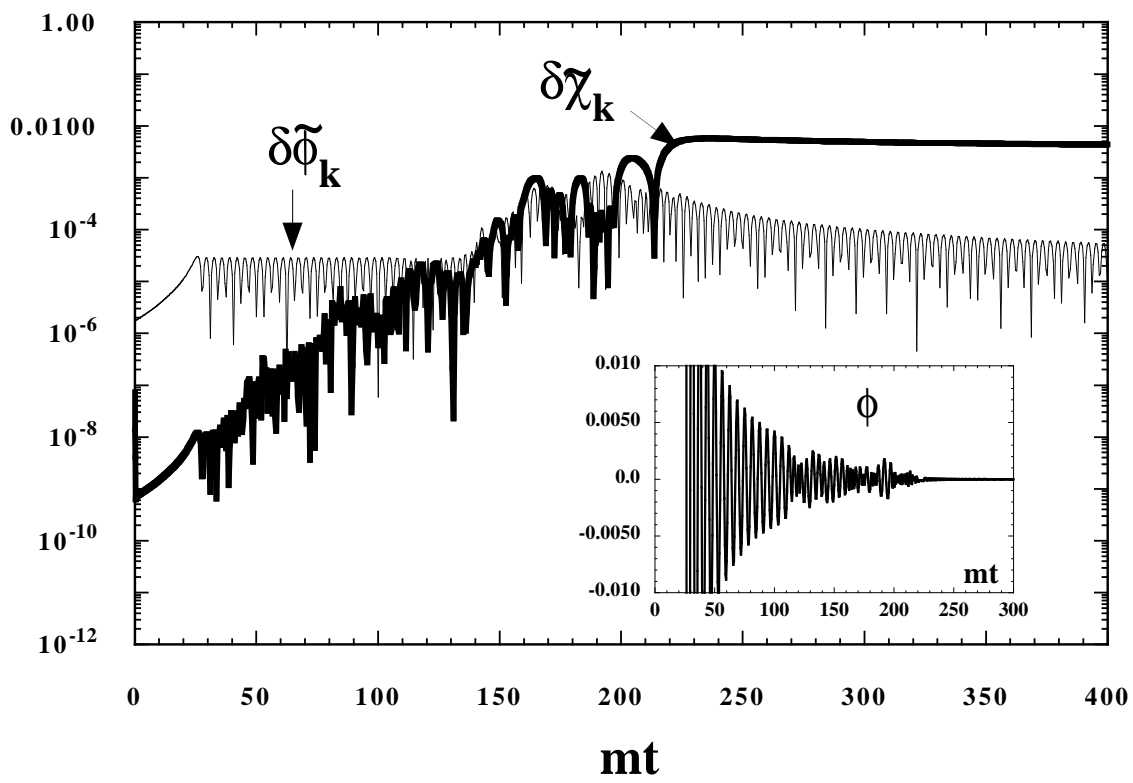

Fig. 6(b)

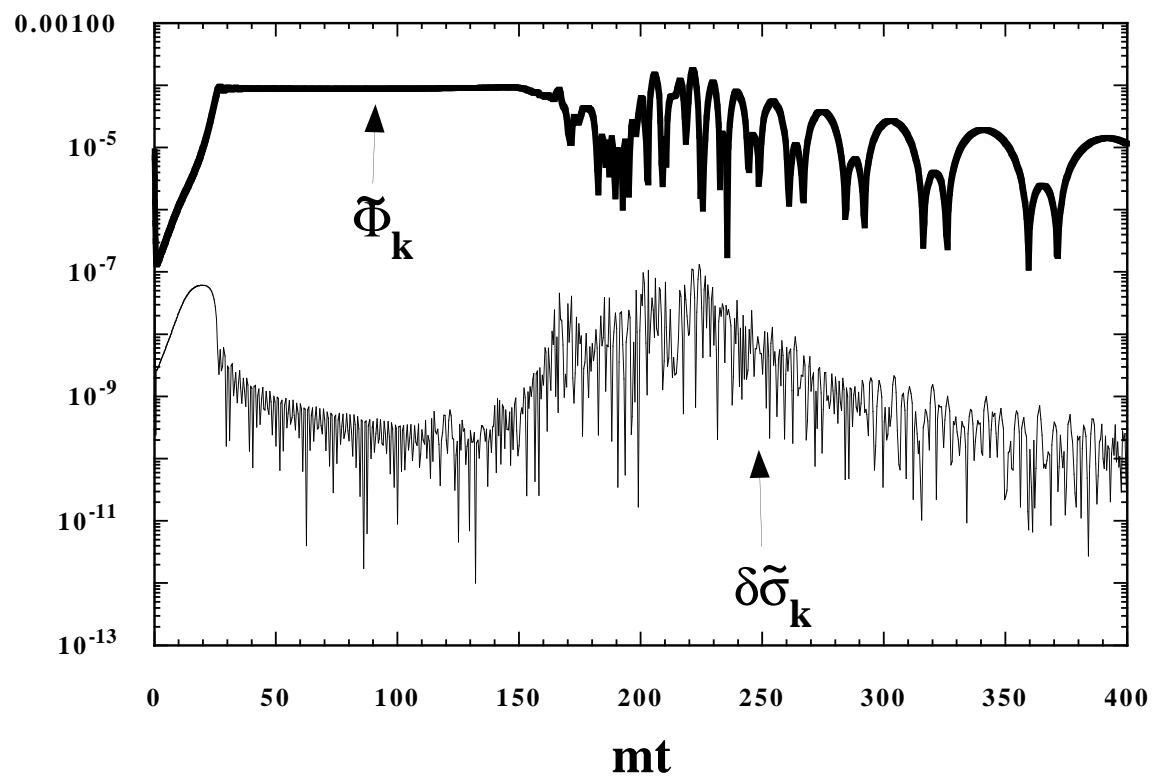

FIG. 6: The evolutions of field perturbations $\delta \tilde{\phi}_{k}, \delta \tilde{\chi}_{k} \delta \tilde{\sigma}_{k}$, and the metric perturbation $\tilde{\Phi}_{k}$ for a super-Hubble mode $\bar{k}=10^{-26}$ with $g=5.0 \times 10^{-4}, \tilde{g} / g=2.0 \times 10^{-2}$, $d=6, b_{*}=1.0 \times 10^{5} / m_{\mathrm{pl}}$, and $m=10^{-6} m_{\mathrm{pl}}$ during inflation and preheating. Inset of Fig. 6(a): The evolution of the inflaton condensate $\phi$.

We have also investigated other values of the coupling $g$, and numerical results exhibit the similar behavior. The 
large scale cosmic microwave background (CMB) anisotropies will not be significantly modified with the existence of the preheating phase, even taking into account the coupling $\tilde{g}^{2} \phi^{3} \chi$. However, the additional enhancement of the super-Hubble metric perturbation found in Fig. 6(b) for $m t \gtrsim 180$ may give some small imprints in the CMB spectrum.

The $\delta \sigma_{k}$ fluctuation in super-Hubble scales can be amplified a little in a short stage as in the case of $\delta \phi_{k}$. In Fig. 6(b), we find that $\delta \sigma_{k}$ increases by about two orders of magnitude during $140 \lesssim m t \lesssim 220$. However, for $g \gtrsim 3.0 \times 10^{-4}$ and values of $\tilde{g}$ which satisfy the condition of (3.39), numerical calculations imply that the growth of super-Hubble $\delta \sigma_{k}$ modes is hardly expected except in the case where $\tilde{g} / g$ is close to its upper bound. Even when $\tilde{g} / g$ is close to its upper bound as in the case of $g=5.0 \times 10^{-4}, \tilde{g} / g=2.0 \times 10^{-2}$, the enhancement is found to be weak. Moreover, final fluctuations $\delta \tilde{\sigma}_{k}$ in super-Hubble modes are typically smaller than $\delta \tilde{\phi}_{k}$ and $\delta \tilde{\chi}_{k}$ as is found in Fig. 6 .

\section{Fig. 7}

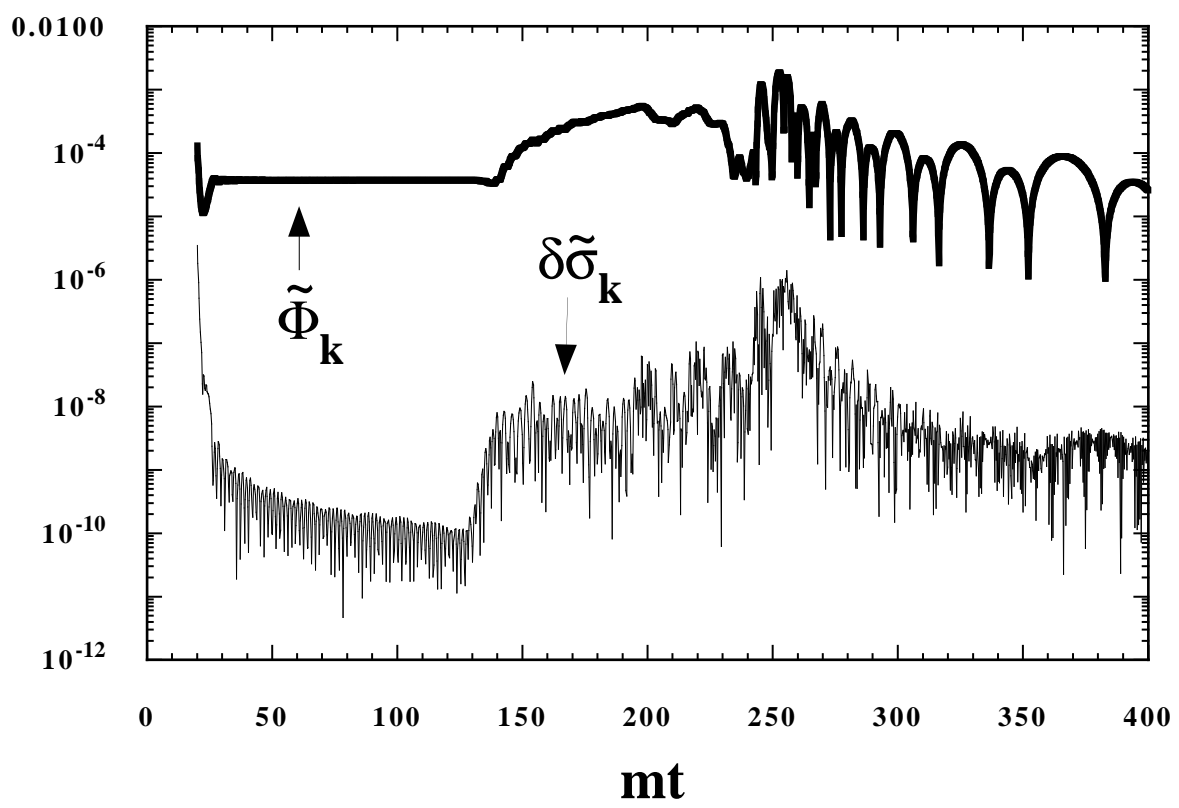

FIG. 7: The evolutions of the metric perturbation $\Phi_{k}$ and the field perturbation $\delta \sigma_{k}$ for a sub-Hubble mode $\bar{k}=5$ with $g=5.0 \times 10^{-4}, \tilde{g} / g=2.0 \times 10^{-2}, d=6$, $b_{*}=1.0 \times 10^{5} / m_{\mathrm{pl}}$, and $m=10^{-6} m_{\mathrm{pl}}$.

As for sub-Hubble scales, amplifications of metric perturbations and the $\delta \sigma_{k}$ fluctuation are relevant compared with the super-Hubble case. In Fig. 7 , we show the evolutions of $\Phi_{k}$ and $\delta \sigma_{k}$ during preheating for a sub-Hubble mode $\bar{k}=5$ with $g=5.0 \times 10^{-4}, \tilde{g} / g=2.0 \times 10^{-2}$. In this case, the sub-Hubble fluctuation of $\delta \tilde{\chi}_{k}$ is larger than in the super-Hubble case at the start of preheating. We find in Fig. 7 that $\Phi_{k}$ increases by more than one order of magnitude, which indicates that metric preheating can be vital in small scales in the presence of the $\tilde{g}^{2} \phi^{3} \chi$ term. The dilaton fluctuation in sub-Hubble modes is also enhanced with the growth of metric perturbations. However, the final $\delta \sigma_{k}$ fluctuation does not exceed its fluctuation at the onset of preheating. If we choose smaller values of $\tilde{g}$, the enhancement of sub-Hubble $\Phi_{k}$ and $\delta \sigma_{k}$ modes becomes weaker. For the couplings which range $3 \times 10^{-4} \lesssim g \lesssim 10^{-2}$, we numerically find that the sub-Hubble dilaton fluctuation is not relevantly amplified for the value of $\tilde{g}$ which is smaller by one order of magnitude than its upper bound given by Eq. (3.39). When the standard coupling $g^{2} \phi^{2} \chi^{2}$ 
dominates over the coupling $\tilde{g}^{3} \phi^{3} \chi$ (namely $g \gg \tilde{g}$ ), we conclude that the fluctuation of dilaton can be held static both in the sub- and super-Hubble scales.

\section{CONCLUDING REMARKS AND DISCUSSIONS}

In this paper, we have studied preheating after inflation with a quadratic inflaton potential $V(\phi)=\frac{1}{2} m^{2} \phi^{2}$ in the presence of a dilaton field $\sigma$ which represents the scale of compactifications in a higher-dimensional generalized Kaluza-Klein theory. We consider the Candelas-Weinberg model where extra dimensions are compactified on the sphere with a cosmological constant and a one-loop quantum correction (Casimir effects). In the chaotic inflation model, a potential barrier which prevents the growth of the internal space disappears for large values of inflaton. However, the fine-tuned initial conditions and parameters of the model naturally lead to successful inflation. We find that the existence of dilaton during inflation hardly affects the evolution of inflaton, and the chaotic inflationary scenario proceeds in the usual manner as long as initial conditions are chosen so that dilaton does not go beyond the potential barrier.

At the stage of preheating after inflation, another scalar field $\chi$ coupled to inflaton can be amplified by parametric resonance due to the oscillation of inflaton. In addition to the standard coupling $\frac{1}{2} g^{2} \phi^{2} \chi^{2}$, we have also included the coupling $\tilde{g}^{2} \phi^{3} \chi$ by which the exponential suppression of the super-Hubble $\chi$ fluctuation can be avoided during inflation. We include metric perturbations explicitly for scalar field equations, and investigate how the fluctuation of dilaton will be amplified both in super- and sub-Hubble scales. Neglecting metric perturbations, the equation for the dilaton fluctuation is reduced to the form of Mathieu equation as in the case of the $\chi$ fluctuation at the linear stage of preheating. Since the resonance parameter $q$ is smaller than unity during the whole stage of preheating, the dilaton fluctuation does not exhibit parametric amplification in the rigid spacetime case.

In the perturbed metric case, it is generally expected that field resonances will stimulate the enhancement of metric perturbations $\Phi_{k}$. In the case of $\tilde{g}=0$, however, since low momentum modes of the $\chi$ field fluctuation are severely suppressed during inflation, super-Hubble metric perturbations are hardly affected by parametric amplification of the field perturbation. We have numerically verified that super-Hubble metric perturbations remain almost constant during preheating, and also found that the dilaton fluctuation in super-Hubble modes can not be enhanced. As for sub-Hubble modes with $\tilde{g}=0$, the $\chi$ field fluctuation is not suppressed relative to super-Hubble modes. However, since the source term in the equation of $\Phi_{k}$ contains the time derivative of the homogeneous $\chi$ field, we can not expect the strong amplification of dilaton fluctuation as well as metric perturbations.

If the coupling $\tilde{g}^{2} \phi^{3} \chi$ is taken into account, both the homogeneous and super-Hubble fluctuational parts of the $\chi$ field can escape the inflationary suppression. These are about $(\tilde{g} / g)^{2}$ times those of inflaton at the onset of preheating. In this case, the $\chi$ fluctuation can typically increase to the order of the inflaton fluctuation, after which the backreaction effect of created $\chi$ particles onto the $\phi$ field begins to be relevant. This restricts the further excitement of the $\chi$ fluctuation, and amplifications of the super-Hubble metric perturbation and the dilaton fluctuation are found to be weak even for large values of $\tilde{g}$ which are close to its upper bound. As for sub-Hubble scales, the dilaton fluctuation can be modestly amplified by the growth of metric perturbations. However, the dilaton fluctuation does not grow for 
the value of $\tilde{g}$ which is smaller by one order of magnitude than its upper bound given by Eq. (3.39). We argue that the stability of compactifications can be preserved during preheating in the quadratic chaotic inflationary scenario as long as $\tilde{g} \ll g$.

We have found that the enhancement of the dilaton perturbation is intimately related with the generation of metric perturbations. Although we only considered the backreaction due to field fluctuations, we should also include second order metric perturbations to the background equations for a consistent study. In fact, the effective momentum tensor formalism in Ref. [44] gives rise to the coupling of the metric $\Phi$ and the fluctuation $\delta \chi_{k}$ in the equation of the homogeneous $\phi$ field, which leads to the growth of the super-Hubble $\delta \chi_{k}$ fluctuation as well as the homogeneous $\chi$ field. Although it is expected that metric perturbations in super-Hubble scales remain well within linear regimes in the case of $\tilde{g}=0$ as in Ref. [41], inclusion of second order metric backreaction effects may lead to the distortion of the large-scale CMB spectrum as well as the enhancement of sub-Hubble metric and field fluctuations in the presence of the interaction $\tilde{g}^{2} \phi^{3} \chi$. In addition to this, rescattering effects of field and metric fluctuations (i.e. mode-mode coupling) will be important at the final stage of preheating [16,21,22, 45]. Although we do not consider these complicated issues in this paper, we should take into account the full backreaction and the rescattering effects for a complete study of preheating.

In this paper, we have restricted ourselves to the massive inflaton model as a first step toward understanding the dynamics of preheating with extra dimensions. We found that amplification of the super-Hubble dilaton fluctuation is weak in this model. However, in other models of inflation, there may be a possibility that extra dimensions will be unstable during preheating. Indeed, it was recently suggested that super-Hubble metric perturbations can be strongly enhanced in broad classes of models [34, 35, 37]. One of such models is the massless chaotic inflation model $V(\phi)=\frac{1}{4} \lambda \phi^{4}$ with the interaction $\frac{1}{2} g^{2} \phi^{2} \chi^{2}$. In this model, even when the effective mass of the $\chi$ field is comparable to the Hubble expansion rate $H$, the super-Hubble $\chi$ field fluctuation can be excited by parametric resonance. This will lead to the enhancement of the dilaton fluctuation in long wave modes through the amplification of super-Hubble metric perturbations. It was also pointed out in Ref. [35] that the negative coupling $\frac{1}{2} g^{2} \phi^{2} \chi^{2}$ with $g^{2}<0$ 46] or the negative nonminimal coupling $\frac{1}{2} \xi R \chi^{2}$ with $\xi<0$ 4 will escape the inflationary suppression of the super-Hubble $\chi$ fluctuation. In addition to this, although we did not consider the interaction between $\sigma$ and $\chi$ fields, including this coupling may strengthen parametric resonance of scalar fields 48. It is quite interesting to investigate the evolution of metric and field fluctuations in broad classes of models with several interactions in the sense that we can constrain the inflaton potential in terms of distortions from the CMB spectrum.

Although we have investigated compactifications on the sphere, there exist several ways of compactifications on other manifolds. For example, the dilaton does not have its own potential in the torus compactification, which would lead to the growth of extra dimensions during inflation as was analyzed in Ref. [12]. In this case, since the resonance term $g^{2} \phi^{2} e^{-d \sigma / \sigma_{*}}$ in the l.h.s. of Eq. (3.8) will be suppressed during inflation, the ordinary picture of preheating may be modified in the presence of extra dimensions. It is also of interest to investigate the dynamics of inflation and preheating in more realistic models of compactifications such as Calabi-Yau manifolds, because it is possible to judge whether such compactifications are appropriate or not from the cosmological point of view. These issues are under consideration. 


\section{ACKNOWLEDGMENTS}

The author would like to thank Bruce A. Bassett, Kei-ichi Maeda, Shinji Mukohyama, Takashi Torii, Kunihito Uzawa, Fermin Viniegra, and Hiroki Yajima for useful discussions and comments. This work was supported partially

by a Grant-in-Aid for Scientific Research Fund of the Ministry of Education, Science and Culture (No. 09410217), and by the Waseda University Grant for Special Research Projects.

[1] M. B. Green, J. H. Schwartz, and E. Witten, Superstring theory (Cambridge University Press, Cambridge, England, 1987).

[2] P. Horava and E. Witten, Nucl. Phys. B460, 506 (1996).

[3] L. Randall and R. Sundrum, Phys. Rev. Lett. 83, 3370 (1999); L. Randall and R. Sundrum, hep-th/9906064.

[4] M. Visser, Phys. Lett. 159B, 22 (1985); hep-th/9910093; V.A. Rubakov and M.E. Shaposhnikov, Phys. Lett. 125B, 139 (1983).

[5] I. Antoniadis, Phys. Lett. 246B, 377 (1990); N. Arkani-Hamed, S. Dimopoulos, and G. Dvali, Phys. Lett. 429B, 263 (1998); I. Antoniadis, N. Arkani-Hamed, S. Dimopoulos, and G. Dvali, Phys. Lett. 436B, 257 (1998).

[6] S. Weinberg, Phys. Lett. 125B, 265 (1983); P. Candelas and S. Weinberg, Nucl. Phys. B237, 397 (1984).

[7] D. Bailin, A. Love, and C. E. Vayonakis, Phys. Lett. 142B, 344 (1984).

[8] K. Maeda, Class. Quantum. Grav. 3, 233 (1986); ibid, 344 (1986).

[9] K. Maeda, Phys. Lett. 186B, 33 (1987).

[10] E. W. Kolb and M. S. Turner, The Early Universe (Addison-Wesley, Redwood City, California, 1990); A. D. Linde, Particle Physics and Inflationary Cosmology (Harwood, Chur, Switzerland, 1990).

[11] L. Amendola, E. W. Kolb, M. Litterio, and F. Occhionero, Phys. Rev. D 42, 1944 (1990).

[12] A. L. Berkin and K. Maeda, Phys. Rev. D 44, 1691 (1991).

[13] D. H. Lyth, Phys. Lett. B448, 191 (1999); N. Kaloper and A. Linde, Phys, Rev. D 59, 101303 (1999); G. Dvali and S. H. Tye, Phys. Lett. B450, 72 (1999); A. Mazumdar, Phys. Lett. B469, 55 (1999).

[14] J. Traschen and R. H. Brandenberger, Phys. Rev. D 42, 2491 (1990); Y. Shtanov, J. Trashen, and R. H. Brandenberger, Phys. Rev. D 51, 5438 (1995); A. D. Dolgov and D. P. Kirilova, Sov. Nucl. Phys. 51, 273 (1990).

[15] L. Kofman, A. Linde, and A. A. Starobinsky, Phys. Rev. Lett. 73, 3195 (1994).

[16] L. Kofman, A. Linde, and A. A. Starobinsky, Phys. Rev. D 56, 3258 (1997).

[17] P. B. Greene, L. Kofman, A. Linde, and A. A. Starobinsky, Phys. Rev. D 56, 6175 (1997); D. I. Kaiser, Phys. Rev. D 56 706 (1997).

[18] S. Khlebnikov and I. I. Tkachev, Phys. Lett. B390, 80 (1997).

[19] D. Boyanovsky, H. J. de Vega, R. Holman, D. S. Lee, and A. Singh, Phys. Rev. D 51, 4419 (1995); D. Boyanovsky, D. Cormier, H. J. de Vega, R. Holman, A. Singh, and M. Srednicki, Phys. Rev. D 56, 1939 (1997).

[20] J. Baacke, K. Heitmann, and C. Pätzold, Phys. Rev. D 55, 2320 (1997); ibid D 56, 6556 (1997).

[21] S. Khlebnikov and I. I. Tkachev, Phys. Rev. Lett. 77, 219 (1996).

[22] S. Khlebnikov and I. I. Tkachev, Phys. Rev. Lett. 79, 1607 (1997).

[23] T. Prokopec and T. G. Roos, Phys. Rev. D 55, 3768 (1997).

[24] E. W. Kolb, A. D. Linde, and A. Riotto, Phys. Rev. Lett. 77, 3716 (1996); E. W. Kolb, A. Riotto, and I. I. Tkachev, Phys. Lett. B423, 348 (1998).

[25] S. Khlebnikov, L. Kofman, A. Linde, and I. I. Tkachev, Phys. Rev. Lett. 81, 2012 (1998); I. I. Tkachev, S. Khlebnikov, L. Kofman, A. Linde, Phys. Lett. B440, 262 (1998); S. Kasuya and M. Kawasaki, Phys. Rev. D 58, 083516 (1998).

[26] L. Kofman, A. Linde, and A. A. Starobinsky, Phys. Rev. Lett. 76, 1011 (1996); I. I. Tkachev, Phys. Lett. B376, 35 (1996); S. Kasuya and M. Kawasaki, Phys. Rev. D 56, 7597 (1997).

[27] S. Khlebnikov and I. I. Tkachev, Phys. Rev. D 56, 653 (1997); B. A. Bassett, Phys. Rev. D 56, 3439 (1997); D. Tilley and R. Maartens, gr-qc/0002089.

[28] A. Mazumdar and L. E. Mendes, Phys. Rev. D 60, 103513 (1999).

[29] S. Mukohyama, Phys. Rev. D 57, 6191 (1998); K. Uzawa, Y. Morisawa, and S. Mukohyama, gr-qc/9912108.

[30] S. Tsujikawa, Phys. Rev. D 61, 124002 (2000).

[31] G. G. Ross, Phys. Lett. B171, 46 (1986); G. G. Ross and S. Sarkar, Nucl. Phys. B461, 597 (1996).

[32] H. Kodama and T. Hamazaki, Prog. Theor. Phys. 96, 949 (1996); Y. Nambu and A. Taruya, Prog. Theor. Phys. 97, 83 (1997); F. Finelli and R. Brandenberger, Phys. Rev. Lett. 821362 (1999); M. Parry and R. Easther, Phys. Rev. D 59 061301 (1999). 
[33] B. A. Bassett, D. I. Kaiser, and R. Maartens, Phys. Lett. B455, 84 (1999); B. A. Bassett, F. Tamburini, D. I. Kaiser, and R. Maartens, Nucl. Phys. B561, 188 (1999).

[34] B. A. Bassett and F. Viniegra, hep-ph/9909353, to appear in Physical Review D.

[35] B. A. Bassett, C. Gordon, R. Maartens, and D. I. Kaiser, Phys. Rev. D. 061302 (R) (2000).

[36] S. Tsujikawa and B. A. Bassett, hep-ph/0003068, to appear in Physical Review D.

[37] F. Finelli and R. Brandenberger, hep-ph/0003172.

[38] V. F. Mukhanov, H. A. Feldman, and R. H. Brandenberger, Phys. Rep. 215, 293 (1992).

[39] N. W. Mac Lachlan, Theory and Applications of Mathieu Functions (Dover, New York, 1961).

[40] P. Ivanov, Phys, Rev. D 61, 023505 (2000).

[41] K. Jedamzik and G. Sigl, Phys, Rev. D 61, 023519 (2000).

[42] A. R. Liddle, D. H. Lyth, K. A. Malik, and D. Wands, Phys, Rev. D 61, 103509 (2000).

[43] A. B. Henriques and R. G. Moorhouse, hep-ph/0003141.

[44] L. R. Abramo, R. H. Brandenberger, and V. F. Mukhanov, Phys. Rev. D 56, 3248 (1997).

[45] M. Parry and R. Easther, hep-ph/9910441.

[46] B. R. Greene, T. Prokopec, and T. G. Roos, Phys. Rev. D 56, 6484 (1997).

[47] As for the particle production by nonminimal coupling, see the following papers: B. A. Bassett and S. Liberati, Phys. Rev. D 58021302 (1998); S. Tsujikawa, K. Maeda, and T. Torii, Phys. Rev. D 60, 063515 (1999); 123505 (1999); Phys. Rev. D 61, 103501 (2000).

[48] B. A. Bassett, Phys. Rev. D 58, 021303 (1998); B. A. Bassett and F. Tamburini, Phys. Rev. Lett. 81, 2630 (1998). 